\title{
Spatial Spectrum-Based Imaging for UWB Through-the-Wall MIMO Arrays
}

\author{
Biying Lu, Xin Sun, Yang Zhao, and Zhimin Zhou \\ College of Electronic Science and Engineering, National University of Defense Technology, Changsha, \\ Hunan 410073, China \\ Correspondence should be addressed to Biying Lu; lubiying@nudt.edu.cn
}

Received 2 April 2014; Revised 23 June 2014; Accepted 28 June 2014; Published 21 July 2014

Academic Editor: Ahmed Shaharyar Khwaja

Copyright (C) 2014 Biying Lu et al. This is an open access article distributed under the Creative Commons Attribution License, which permits unrestricted use, distribution, and reproduction in any medium, provided the original work is properly cited.

To keep the system complexity at a reasonable level and conform to the propagation demands, MIMO arrays are usually sparse in through-the-wall applications, which results in corrupted and gapped data. The corresponding imaging results are seriously affected by the high-level sidelobes. To solve this problem, a new imaging model for ultra-wideband (UWB) MIMO arrays is constructed via spatial spectrum theory in this paper. Based on the model, the characteristics of the spatial spectrum for the MIMO array and its effects on imaging are discussed. To improve the imaging quality, a through-the-wall imaging enhancement method is proposed via spatial spectrum estimation. Synthetic and experimental results show that, unlike the conventional amplitude weighting methods and nonlinear techniques, the proposed method can efficiently suppress sidelobes in the imagery, especially for the sparse MIMO array, and consequently improve the target image quality without degrading the mainlobe resolution. The proposed method has been successfully used in our real through-the-wall radar system.

\section{Introduction}

Ultra-wideband (UWB) through-the-wall imaging (TWI) approaches that can detect objects through obstacles, such as walls, doors, and other opaque materials, are considered powerful tools for a variety of civilian and military applications [1-5].

In TWI applications, the imaging component of the application is considered the most important because it is usually the first step for the subsequent processes, such as detection, identification, and wall parameters estimation [6-10]. Currently, to obtain a satisfying target image, two types of radars are widely used: synthetic aperture radar (SAR) and multiple input multiple output (MIMO) radar. Although SAR has better resolution, it has a heavy time cost. By using the high-speed electronic switch, the time to acquire a dataset in a MIMO system is greatly reduced, compared to SAR systems. Therefore, MIMO radar is preferred over SAR in real applications, especially for moving target imaging.

By using the proper array design method, we can obtain an optimal array configuration. However, in certain real cases, the equipment complexity and the shape may be our first consideration. Therefore, we make the tradeoff between size and performance [11]. For example, to achieve the Nyquist sampling criterion, the interelement space $(d)$ must be kept below half of the wavelength $(\lambda)$ for the MIMO array [12]. However, conforming to this criterion will lead to a large number of array elements, even for a small aperture. Usually, when a MIMO array is used in TWI applications, the element spacing is made significantly higher than $\lambda / 2$ to keep the system complexity at reasonable levels and to increase the element size to achieve an acceptable SNR. Furthermore, for a typical TWI radar system, the most commonly used frequency range is from $1 \mathrm{GHz}$ to $3 \mathrm{GHz}$ to support the range resolution and wall propagation ability. Therefore, for the ultrawideband signal, even if more elements can be placed in the equipment, the elements are usually dense in the low frequency band but sparse in the high frequency band. In such a case, the MIMO array will not be optimal but it will be sparse with gapped virtual elements, which would otherwise diminish the array imaging performance. As a result, the image quality of the TWI results, in real 
applications, is significantly limited by the ratio of the main to sidelobe amplitude.

To suppress the sidelobes and improve the image quality, many imaging methods for through-the-wall imaging, including the back projection (BP) method $[13,14]$, the beamforming method $[15,16]$, and the tomography method $[17,18]$, are presented in recent years. In these methods, the sidelobes are reduced by applying an amplitude weighting function to the data prior to the final IFFT. However, the sidelobes have been reduced at the expense of the main lobe width, which determines the ultimate resolution of the imagery [19]. For example, the Hanning main lobe is twice as wide (null-to-null) as the sinc function. These methods are consequently a compromise between a narrow main lobe (high resolution) and low sidelobes.

To retain the main lobe resolution while reducing the sidelobes, several nonlinear signal processing methods are introduced into radar imaging. Typical methods include spatially variant apodization (SVA), super-SVA, and the CLEAN technique [19-22]. By using interpolation or extrapolation operations, these methods are successfully used in SAR signal data processing to minimize the effects of corrupted and gapped data. However, for MIMO radar, because of the more complicated signal channels, the distribution of the received data is significantly different from that in SAR. In this situation, the performance of these methods is seriously affected.

Based on the rigorous derivation of the UWB MIMO array and experimental validation via real TWI radar systems, we proposed in this paper a through-the-wall imaging enhancement method via spatial spectrum theory. Unlike the conventional amplitude weighting methods and nonlinear techniques, the proposed method can effectively suppress the sidelobes from imagery, especially for the UWB sparse MIMO array, and consequently enhance the target image quality without degrading the main lobe resolution.

This paper is organized as follows. In Section 2, the imaging model for the MIMO array is constructed via spatial spectrum theory. Then, the spatial spectrum of the UWB MIMO array is deeply analyzed. The effects of the spatial spectrum distribution on PSF are discussed, and the spatial spectrum characteristics for the typical TWI UWB MIMO array are obtained. In Section 4, to improve the image quality, an imaging enhancement method by spatial spectrum estimation is proposed. Synthetic and experimental processing results are given in Section 5. Conclusions end this paper.

\section{Spatial Spectrum-Based Imaging Model for a MIMO Array}

We assume a MIMO array has $\mathrm{M}$ transmitters and $\mathrm{N}$ receivers, as shown in Figure 1. For convenience, in the following derivation we choose one transmitter and one receiver, and the polar coordinate origin is assumed at the target center. We assume a stepped frequency waveform as the

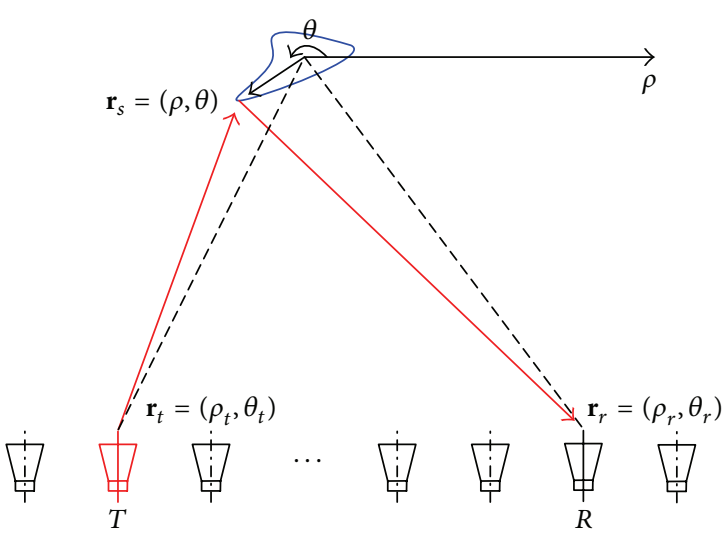

FIGURE 1: The geometry of the MIMO array imaging scene.

transmitted signal, which has a frequency range of $\left[f_{0}, f_{P-1}\right]$ and a frequency increment of $\Delta f$ :

$$
\begin{gathered}
s(t)=\sum_{p=0}^{P-1} e^{j 2 \pi f_{p} t}, \\
f_{p}=\left(f_{0}+p \Delta f\right), \quad p=0,1, \ldots, P-1,
\end{gathered}
$$

where $f_{0}$ is the start frequency. The variable $P$ denotes the frequency numbers.

We consider a general bistatic radar scene with a target as shown in Figure 1. The transmitter located at $\mathbf{r}_{t}=\left(R_{t}, \theta_{t}\right)$ transmits the EM wave. The wave arrives at the reflection point located at $\mathbf{r}_{s}=(\rho, \theta)$ and is backscattered. Under the assumption of free space propagation, the total received backscattered field of the distributed target by the receiver at $\mathbf{r}_{r}=\left(R_{r}, \theta_{r}\right)$ can be expressed as

$$
G\left(f_{p}, \mathbf{r}_{t}, \mathbf{r}_{r}\right)=\int_{\rho} \int_{\theta} \sigma(\rho, \theta) e^{j 2 \pi\left(f_{p} / c\right)\left(\left|\mathbf{r}_{t}-\mathbf{r}_{s}\right|+\left|\mathbf{r}_{r}-\mathbf{r}_{s}\right|\right)} d \rho d \theta,
$$

where $\sigma(\rho, \theta)$ is the reflection function of the target. Assuming $\rho \ll \rho_{t}$ and $\rho \ll \rho_{r}$, the following approximation holds:

$$
\begin{aligned}
& \left|\mathbf{r}_{t}-\mathbf{r}_{s}\right| \approx \rho_{t}-\rho \cos \left(\theta-\theta_{t}\right), \\
& \left|\mathbf{r}_{r}-\mathbf{r}_{s}\right| \approx \rho_{r}-\rho \cos \left(\theta-\theta_{r}\right) .
\end{aligned}
$$

Then, (2) becomes

$$
\begin{aligned}
G\left(f_{p}, \mathbf{r}_{t}, \mathbf{r}_{r}\right) \\
=e^{j 2 \pi\left(f_{p} / c\right)\left(\rho_{t}+\rho_{r}\right)} \\
\quad \times \int_{\rho} \int_{\theta} \sigma(\rho, \theta) e^{-j 2 \pi\left(f_{p} / c\right)\left[\rho \cos \left(\theta-\theta_{t}\right)+\rho \cos \left(\theta-\theta_{r}\right)\right]} d \rho d \theta .
\end{aligned}
$$


The fixed value $e^{j 2 \pi\left(f_{p} / c\right)\left(\rho_{t}+\rho_{r}\right)}$ outside the integrals can be neglected. We define $x=\rho \cos \varphi, y=\rho \sin \varphi$ in the equivalent Descartes coordinates and the wavenumber $k=2 \pi f_{p} / c$ in the expression. Then, we rewrite (2) in the Descartes coordinate system:

$$
\begin{aligned}
& G\left(k, \mathbf{r}_{t}^{\prime}, \mathbf{r}_{r}^{\prime}\right) \\
& =\int_{x} \int_{y} \sigma(x, y) e^{-j k\left[\left(\cos \theta_{t}+\cos \theta_{r}\right) x+\left(\sin \theta_{t}+\sin \theta_{r}\right) y\right]} d x d y \\
& =\int_{x} \int_{y} \sigma(x, y) e^{-j\left[\left(k_{t, x}+k_{r, x}\right) x+\left(k_{t, y}+k_{r, y}\right) y\right]} d x d y,
\end{aligned}
$$

where $\mathbf{r}_{t}^{\prime}$ and $\mathbf{r}^{\prime}$ denote the positions of the transmitter and receiver, respectively, in Descartes coordinates. $k_{t, x}=k \cos \theta_{t}$ and $k_{t, y}=k \sin \theta_{t}$ are the transmitter wavenumbers in the directions of the $x$-axis and $y$-axis. $k_{r, x}=k \cos \theta_{r}$ and $k_{r, y}=$ $k \sin \theta_{r}$ are the receiver wavenumbers in the directions of the $x$-axis and $y$-axis.

We further define the virtual wavenumbers $k_{v, x}=k_{t, x}+$ $k_{r, x}$ and $k_{v, y}=k_{t, y}+k_{r, y}$. Then, the spatial spectrum $G\left(k_{v, x}, k_{v, y}\right)$ can be expressed as the 2D Fourier transform of the target reflection function, $\sigma(x, y)$

$$
G\left(k_{v, x}, k_{v, y}\right)=\int_{x} \int_{y} \sigma(x, y) e^{-j\left(k_{v, x} x+k_{v, y} y\right)} d x d y .
$$

If the wavenumbers are expressed in the form of vectors as $\mathbf{k}_{v}=\left[k_{v x}, k_{v, y}\right]^{T}, \mathbf{k}_{t}=\left[k_{t, x} k_{t, y}\right]^{T}$, and $\mathbf{k}_{r}=\left[k_{r, x} k_{r, y}\right]^{T}$, then

$$
\mathbf{k}_{v}=\mathbf{k}_{t}+\mathbf{k}_{r}=2 k \cos \frac{\theta_{t}-\theta_{r}}{2}\left[\cos \frac{\theta_{t}+\theta_{r}}{2} \sin \frac{\theta_{t}+\theta_{r}}{2}\right]^{T} .
$$

Here, the vector $\mathbf{k}_{v}$ is the wavenumber for the virtual element, which, as shown in Figure 2, is the vector sum of the transmitter wavenumber $\mathbf{k}_{t}$ and the receiver wavenumber $\mathbf{k}_{r}$.

Therefore, by taking the inverse Fourier transform of the spatial spectrum of the target scattering, that is, $G_{M}\left(k_{x}, k_{y}\right)$, the target image can be obtained:

$$
I(x, y)=\int_{k_{x}} \int_{k_{y}} G_{M}\left(k_{x}, k_{y}\right) \exp \left[j\left(k_{x} x+k_{y} y\right)\right] d k_{x} d k_{y} .
$$

As a result, for the MIMO array constructed by $\mathrm{M}$ transmitters and $\mathrm{N}$ receivers, the spatial spectrum of the

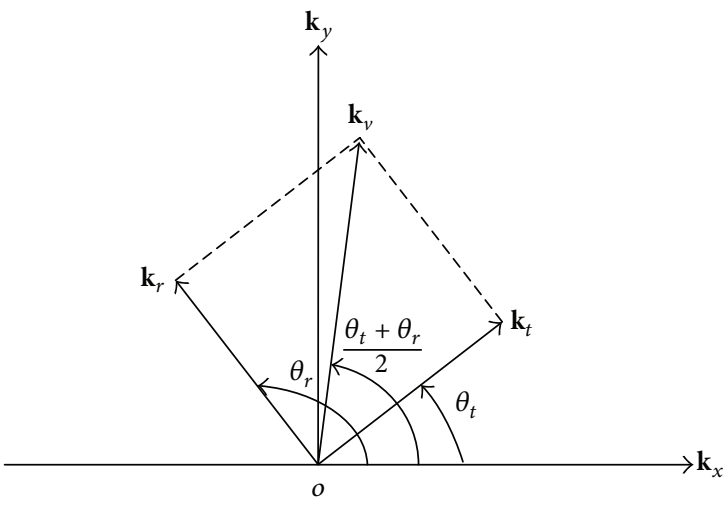

FIGURE 2: The relationship between the virtual element wavenumber, the transmitter wavenumber, and the receive wavenumber.

received signal is determined by the array structure. By using an ideal point target located at the origin, that is, $\sigma(x, y)=$ $\delta(x) \delta(y)$, the point spread function (PSF) of the array system can be demonstrated by (9) in the spatial spectrum domain:

$$
P_{M}\left(k_{x}, k_{y}\right)= \begin{cases}1, & \left(k_{x}, k_{y}\right) \in \mathbf{K} \\ 0, & \left(k_{x}, k_{y}\right) \notin \mathbf{K},\end{cases}
$$

where $\mathbf{K}$ is the support area of the spatial spectrum. After the inverse Fourier transform is performed, the spatial expression of the PSF is

$$
\begin{aligned}
\operatorname{PSF}_{M}(x, y)=\int_{k_{x}} & \int_{k_{y}} P_{M}\left(k_{x}, k_{y}\right) \\
& \times \exp \left[j 2 \pi\left(k_{x} x+k_{y} y\right)\right] d k_{x} d k_{y} .
\end{aligned}
$$

According to (9) and (10), for a MIMO imaging system, the PSF is equivalent to the support area of the spatial spectrum, and the performance of the imaging system can be depicted by the support area of the spatial spectrum.

\section{Spatial Spectrum Analysis for the UWB MIMO Array}

As we know, an imaging system can be fully characterized by the point spread function (PSF) defined as the response of the imaging system to an ideal point source. Equation (10) presents the relationship between the spatial spectrum distribution and the PSF. In this section, the effects of the spatial spectrum distribution on PSF are first discussed. Then, the spatial spectrum of the typical through-the-wall UWB MIMO array is analyzed. 


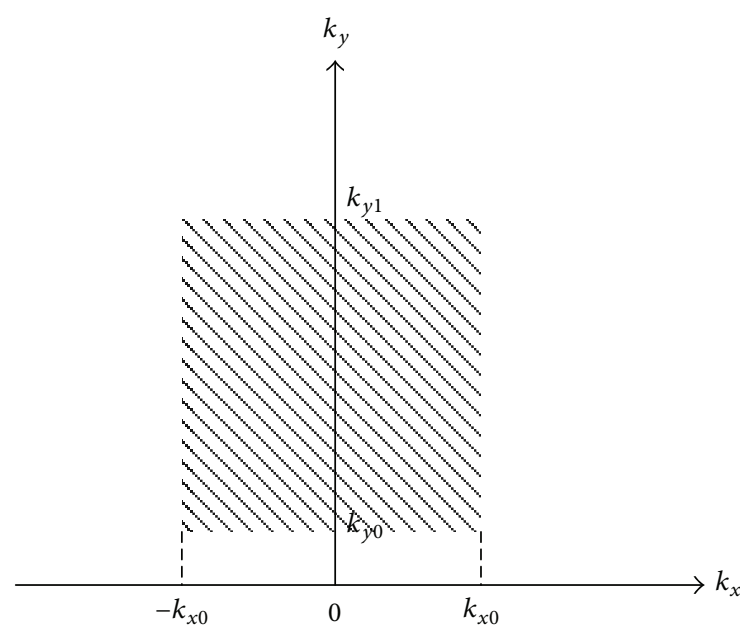

(a) Rectangle

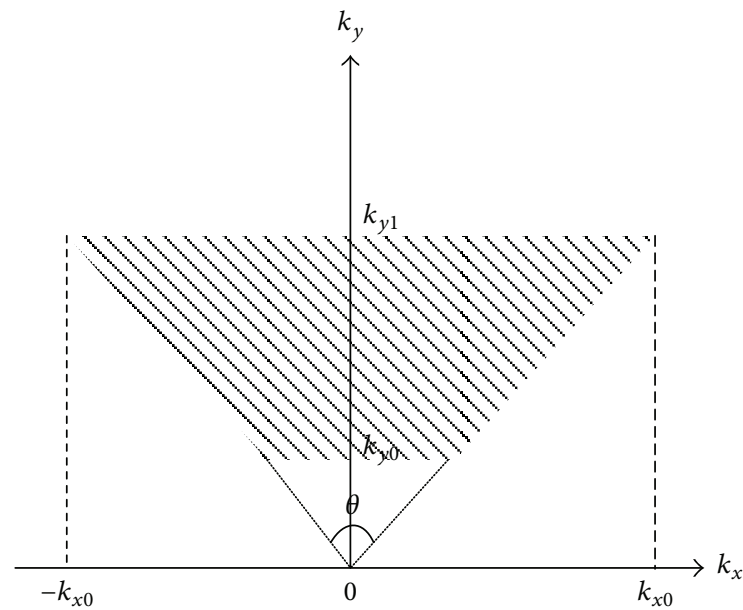

(b) Isosceles trapezoid

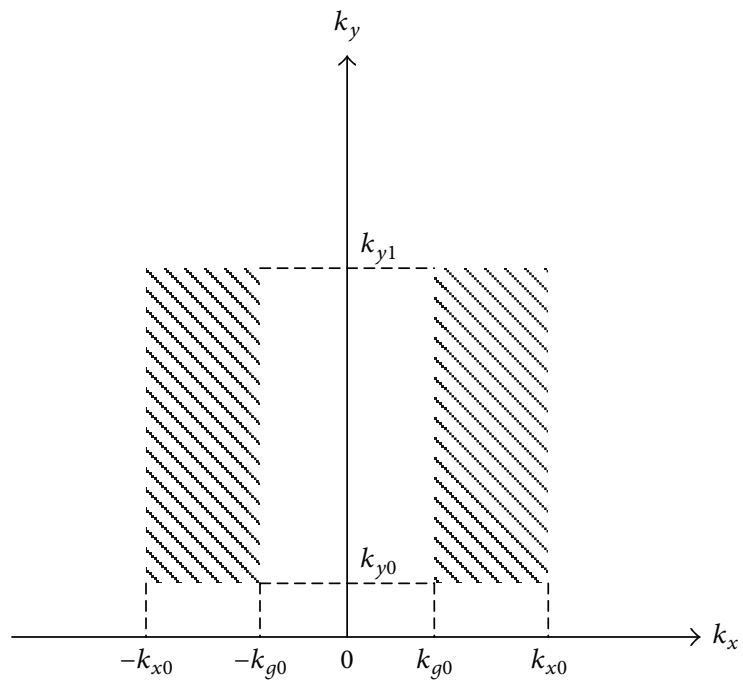

(c) Gapped rectangle

FIGURE 3: Geometric shapes of the spatial spectrum support area.

3.1. Effects of the Spatial Spectrum Distribution on the PSF. For an imaging system, the ideal support area of the spatial spectrum is an evenly sampled rectangle, as shown in Figure 3(a). The corresponding PSF is

$$
\begin{aligned}
\operatorname{PSF}_{r}(x, y)= & \int_{-k_{x 0}}^{k_{x 0}} \exp \left(j 2 \pi x k_{x}\right) d k_{x} \\
& \cdot \int_{k_{y 0}}^{k_{y 1}} \exp \left(j 2 \pi y k_{y}\right) d k_{y} \\
= & \phi \frac{\sin \left(\pi \Delta k_{x} x\right)}{\pi x} \frac{\sin \left(\pi \Delta k_{y} y\right)}{\pi y},
\end{aligned}
$$

where $\Delta k_{x}=2 k_{x 0}, \Delta k_{y}=k_{y 1}-k_{y 0}$, and $\phi=e^{j \pi\left(k_{y 1}+k_{y 0}\right) y}$ are the phase term. The resolution of the image is

$$
\begin{aligned}
& \rho_{x}=\frac{1}{\Delta k_{x}}, \\
& \rho_{y}=\frac{1}{\Delta k_{y}} .
\end{aligned}
$$

In this situation, the highest level of the sidelobe for the sinc function is $-13.3 \mathrm{~dB}$, and the sidelobes are orthogonal and spread along the $x$ - and $y$-axes (see Figure 4(d)).

Unfortunately, the support area of a wideband wide beam imaging system is an annulus sector. In this situation, a trapezoid is used instead of a rectangle to approximate the actual spectral support area to increase the resolution $[23,24]$. Furthermore, the support area of the acquired 


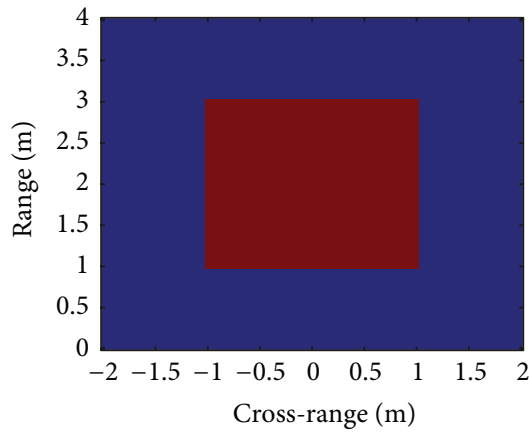

(a)

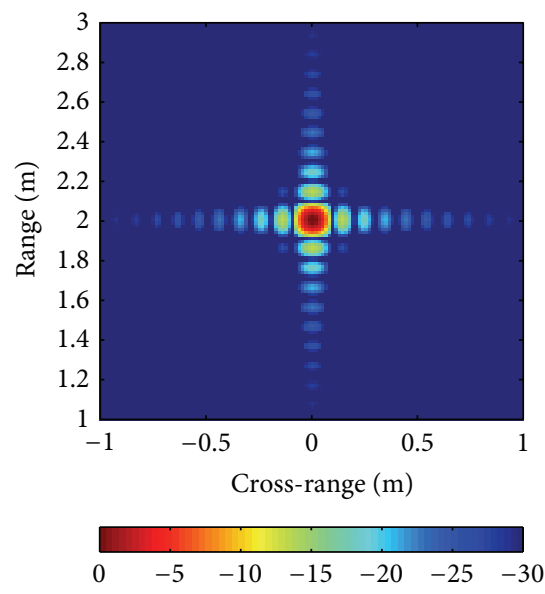

(d)

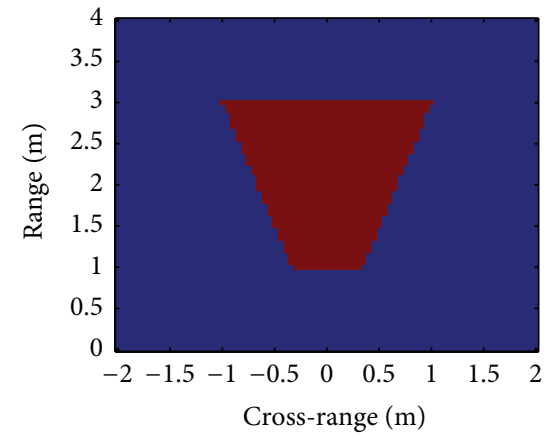

(b)

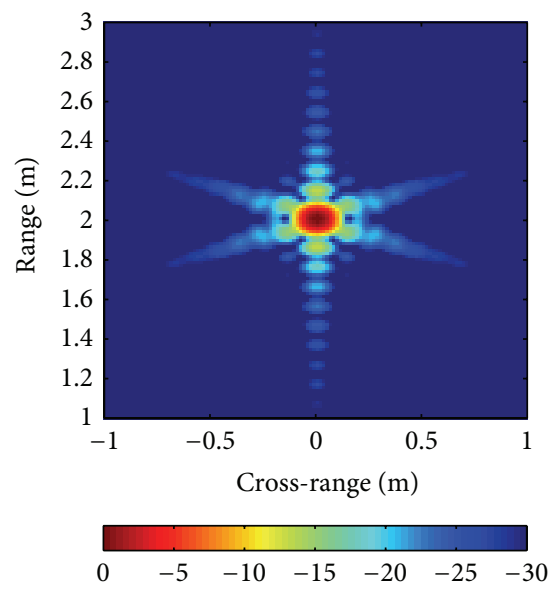

(e)

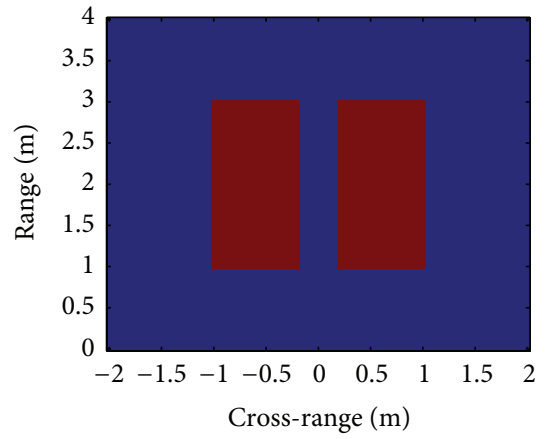

(c)

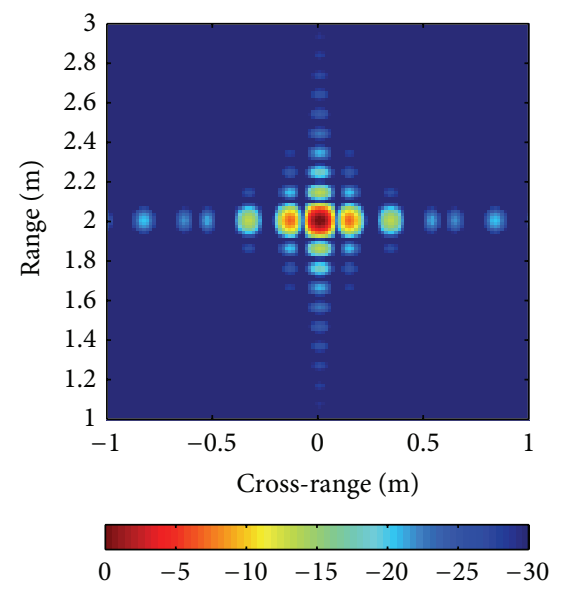

(f)

FIGURE 4: Three different support areas of the spatial spectrum and the corresponding target images: (a) rectangle, (b) isosceles trapezoid, (c) gapped rectangle, (d) target image for Figure 4(a), (e) target image for Figure 4(b), and (f) target image for Figure 4(c).

spectrum is often not continuous due to incomplete data. In radar imaging, a target may be illuminated by the radar from several separate angular regions to improve crossrange resolution, and, consequently, the sampled data will have missing columns $[25,26]$. For the imaging system using fixed aperture, sparse arrays rather than filled arrays are preferred to reduce the number of array elements, which means that spatial samples can more easily be missed $[22,27,28]$.

For the isosceles trapezoid shown in Figure 3(b), the PSF is given as

$$
\begin{aligned}
& \operatorname{PSF}_{t}(x, y) \\
& =\int_{k_{y 0}}^{k_{y 1}} \int_{-k_{y} \tan \theta / 2}^{k_{y} \tan \theta / 2} \exp \left(j 2 \pi x k_{x}\right) \exp \left(j 2 \pi y k_{y}\right) d k_{x} d k_{y} \\
& =\left(\cos \left[\pi\left(k_{y 1}+k_{y 0}\right)\left(y+x \tan \frac{\theta}{2}\right)\right]\right. \\
& \left.\quad \times \sin \left[\pi\left(k_{y 1}-k_{y 0}\right)\left(y+x \tan \frac{\theta}{2}\right)\right]\right) \\
& \quad \times\left(\pi x\left(y+x \tan \frac{\theta}{2}\right)\right)^{-1}
\end{aligned}
$$

$$
\begin{aligned}
& +\left(\cos \left[\pi\left(k_{y 1}+k_{y 0}\right)\left(y-x \tan \frac{\theta}{2}\right)\right]\right. \\
& \left.\times \sin \left[\pi\left(k_{y 1}-k_{y 0}\right)\left(y-x \tan \frac{\theta}{2}\right)\right]\right) \\
& \times\left(\pi x\left(y-x \tan \frac{\theta}{2}\right)\right)^{-1},
\end{aligned}
$$

where $\theta$ is the processing angle shown in Figure 3(b). Equation (13) indicates that the sidelobes are nonorthogonal and spread along the linear axes with $y= \pm x \tan \theta / 2$ (see Figure 4(e)).

For the gapped spatial spectrum shown in Figure 3(c), the PSF is given as

$$
\begin{aligned}
& \operatorname{PSF}_{g}(x, y) \\
& =\left(\int_{-k_{x 0}}^{k_{x 0}} \exp \left(j 2 \pi k_{x} x\right) d k_{x}-\int_{-k_{g 0}}^{k_{g 0}} \exp \left(j 2 \pi k_{x} x\right) d k_{x}\right)
\end{aligned}
$$




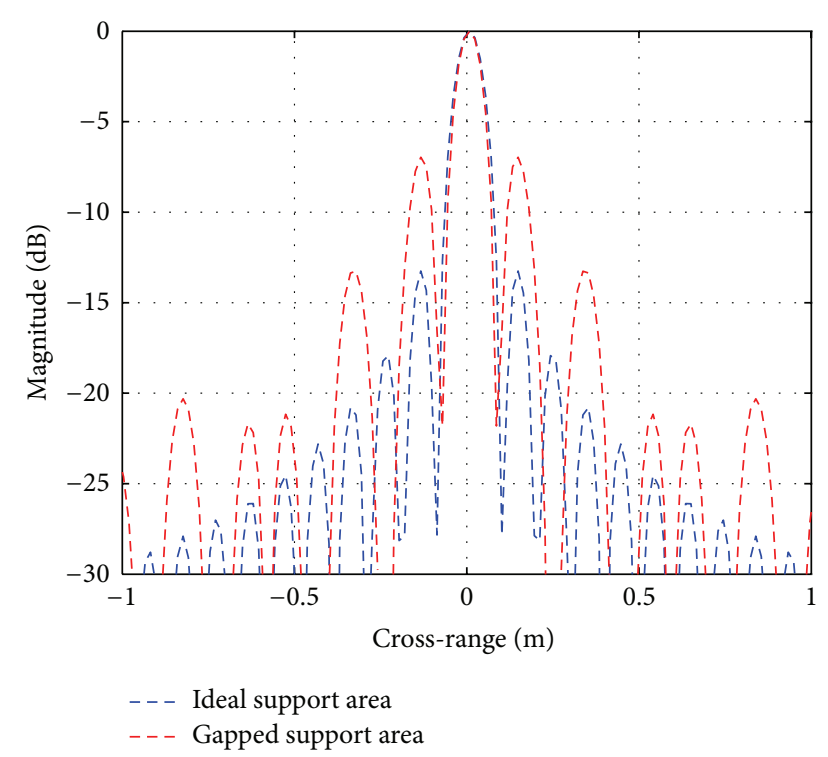

FIGURE 5: The PSF figures in the cross-range direction. In the figure, the blue lines denote the PSF for the ideal support area, that is, Figure 4(a). The red lines denote the PSF for the gapped support area, that is, Figure 4(c).

$$
\begin{aligned}
& \times \int_{k_{y 0}}^{k_{y 1}} \exp \left(j 2 \pi k_{y}\right) d k_{y} \\
= & \frac{\sin \left(2 \pi k_{x 0} x\right)-\sin \left(2 \pi k_{g 0} x\right)}{\pi x} \cdot \frac{\sin \left(2 \pi\left(k_{y 1}-k_{y 0}\right) y\right)}{\pi y} .
\end{aligned}
$$

To analyze the effects of the gapped spatial spectrum on the imaging result, Figure 4 shows three support areas of the spatial spectrum, that is, rectangle, isosceles trapezoid and gapped rectangle, and the corresponding target images. For the rectangle and gapped rectangle support area, the sidelobes are orthogonal and spread along the $x$ - and $y$-axes. When the support area is an isosceles trapezoid, its sidelobes are no longer orthogonal. Additionally, by comparing Figure 4(d) and Figure 4(f), we can find that when the support area is gapped, the sidelobes are significantly enhanced. As a result, the target imaging quality in Figure 4(f) is seriously affected. The PSF figures in the cross-range direction for the ideal support area and the gapped support area are given in Figure 5. The red lines and the blue lines correspond to Figure 4(d) and Figure 4(f), respectively. The main lobe is approximately unchanged, but the sidelobes are significantly different. The highest level of the sidelobes is $-13.3 \mathrm{~dB}$ in the case of ideal support area and $-7.0 \mathrm{~dB}$ when the support area is gapped, as in Figure 4(c).

According to the derivation and figures, the PSF performance is determined by the spatial spectrum distribution. Furthermore, the distribution and levels of the sidelobes are determined by the shape and density of the spatial spectrum, respectively.

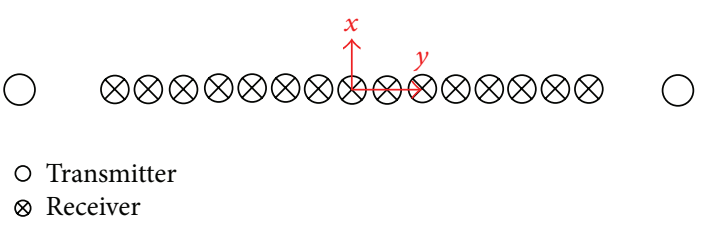

(a) Split transmit aperture array

\section{0}

000000000000000

- Virtual element

(b) Virtual elements

FIGURE 6: Split transmit virtual aperture array and its virtual elements.

3.2. Spatial Spectrum of the Typical TWI UWB MIMO Array. Three characteristics of the spatial spectrum for the UWB MIMO array can be determined. First, a large processing angle is needed in the low frequency band to obtain a satisfying azimuth resolution. However, in real practice, the processing angle is usually limited. Therefore, the support area is no longer nearly rectangular but is described by an annulus sector. In this situation, the trapezoid is usually used instead of the rectangle to approximate the actual spectral support area. Second, to keep the system complexity reasonable, the elements are usually sparse and the element spacing is significantly higher than $\lambda / 2$. For the ultrawideband signal, the spatial spectrum will be dense in the low frequency band and sparse in the high frequency band. Third, for some MIMO arrays with special structures, the corresponding spatial spectrums may be gapped.

In order to explain the characteristics, here we take a spare array, for example. The configuration of the array is shown in Figure 6(a), and the receiver array consists of 15 elements with an interelement spacing of $0.2 \mathrm{~m}$. Two transmit elements are located at $(-2 \mathrm{~m}, 0 \mathrm{~m})$ and $(2 \mathrm{~m}, 0 \mathrm{~m})$, at the ends of the array. The physical aperture length of the array is $4 \mathrm{~m}$. Using the concept of a virtual phase center, a virtual aperture with 30 virtual transmit/receive (T/R) elements is synthesized, as shown in Figure 6(b). The interelement spacing of the synthesized virtual aperture is $0.1 \mathrm{~m}$ except for a gap of $0.6 \mathrm{~m}$ at the midpoint, synthesizing a total aperture length of $3.4 \mathrm{~m}$. The gap at the midpoint is due to the separation of the transmit elements.

The transmitted signal is a stepped frequency waveform, with a range from $0.5 \mathrm{GHz}$ to $1 \mathrm{GHz}$. The increment frequency is $2 \mathrm{MHz}$. For a point target located at $(0 \mathrm{~m}, 5 \mathrm{~m})$, we obtain the spatial spectrum support area shown in Figure 7. There are 30 dotted lines in the figure, corresponding to the 30 virtual elements, and the envelope of the support area is an isosceles trapezoid. For each dotted line, the points are dense when $k_{y}$ is small (corresponding to low frequency) and sparse when $k_{y}$ is large (corresponding to high frequency). Unfortunately, the support area is missing in the middle region, which is caused by the gap in the virtual elements.

To analyze the effects of the gapped spatial spectrum on target imaging, the result and profile in the cross-range direction are presented in Figures 8 and 9, respectively. The 


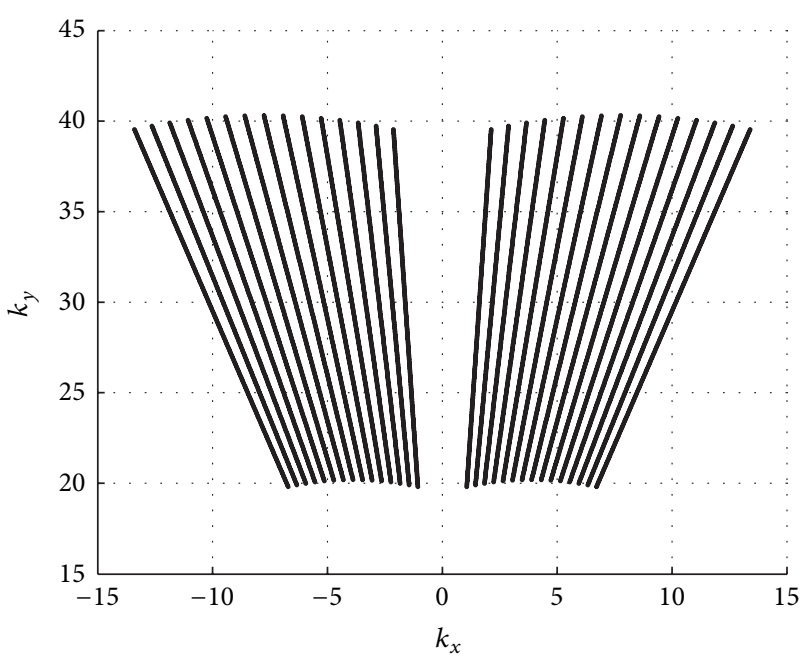

FIGURE 7: Support area of the spatial spectrum for this array.

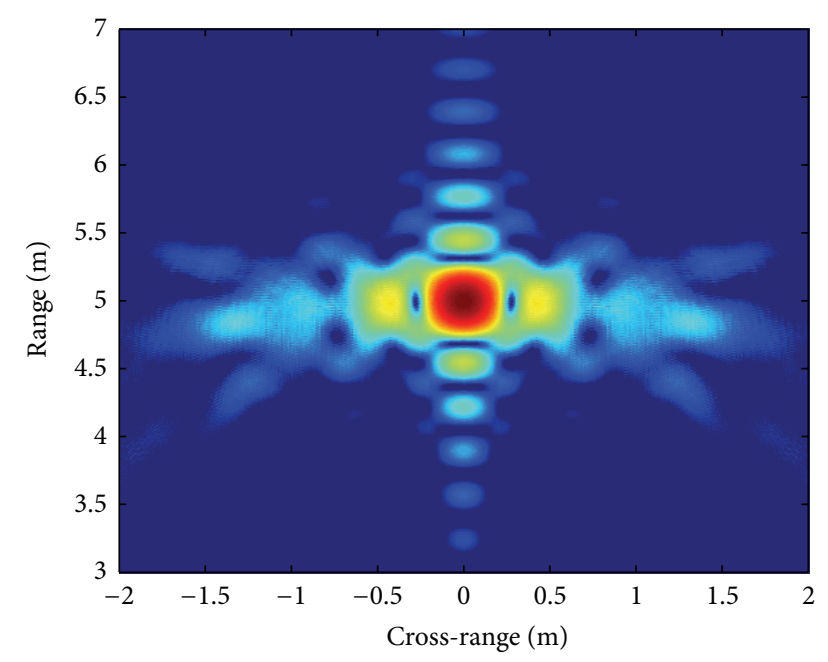

FIGURE 8: The target imaging result.

whole target image is severely affected, and the highest level of the sidelobes is $-10.67 \mathrm{~dB}$.

\section{Through-the-Wall Imaging Enhancement via Spatial Spectrum Estimation}

As shown in the analysis above, the serious sidelobes problem in the STVA system is caused by the missing spatial spectrum. To suppress the sidelobes, conventional methods are applied using a weighting function, such as the Hanning, Hamming, or Blackman functions. However, these weighting methods suppress the sidelobes at the expense of the main lobe resolution. A through-the-wall imaging enhancement method via a spatial spectrum estimation for suppressing the sidelobes without degrading the main lobe resolution is presented in this section.

The principle of our method is to extrapolate the missing support area using the existing spatial spectrum. Then,

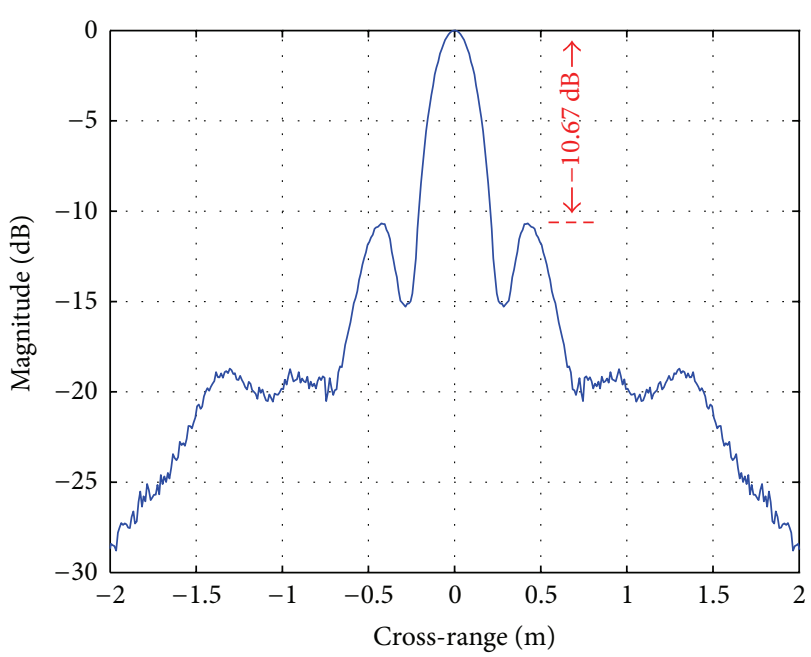

FIGURE 9: The profile in the cross-range direction of the target image.

according to the filled support area, the sidelobes-suppressed image can be obtained by applying the two-dimensional inverse Fourier transform.

The common method for obtaining the support area of the spatial spectrum for the target image is the Stolt interpolation, which is widely used in far-field-based imaging. However, in TWI MIMO radar applications, the distribution of the spatial spectrum is so complicated that the interpolation processing is inaccurate. According to the derived relationship between the image and the spatial spectrum, in our method, the support area is obtained by taking the $2 \mathrm{D}$ Fourier transform of the image. Thus, the steps of our method are as follows.

Step 1. Perform the imaging processing for the received echo. Here, we take the BP imaging method as an example. In the imaging operation, the region of interest is divided into a finite number of pixels in the range and cross-range directions. The complex amplitude image value for the pixel located at $\mathbf{x}=(x, y)$ is obtained by applying frequencydependent phases and weights to all the received data:

$$
\begin{aligned}
I(x, y)=\iiint w \cdot S\left(f_{m}, \mathbf{x}_{T}, \mathbf{x}_{R}\right) \\
\cdot \exp \left(\frac{j 2 \pi f_{m} r\left(\mathbf{x}_{T}, \mathbf{x}, \mathbf{x}_{R}\right)}{c}\right) d \mathbf{x}_{T} d \mathbf{x}_{R} d f_{m}
\end{aligned}
$$

where $w$ is the weighting function to shape the beam. $S(\cdot)$ is the received echo. $\mathbf{x}_{T}$ and $\mathbf{x}_{R}$ denote the transmitter and receiver positions. $r\left(\mathbf{x}_{T}, \mathbf{x}, \mathbf{x}_{R}\right)$ is the compensation signal distance for pixel $\mathbf{x}$, which is computed as follows:

$$
\begin{aligned}
r\left(\mathbf{x}_{T}, \mathbf{x}_{q}, \mathbf{x}_{R}\right)= & \sqrt{\left(x_{T}-x_{q}\right)^{2}+\left(y_{T}-y_{q}\right)^{2}} \\
& +\sqrt{\left(x_{q}-x_{R}\right)^{2}+\left(y_{q}-y_{R}\right)^{2}} .
\end{aligned}
$$




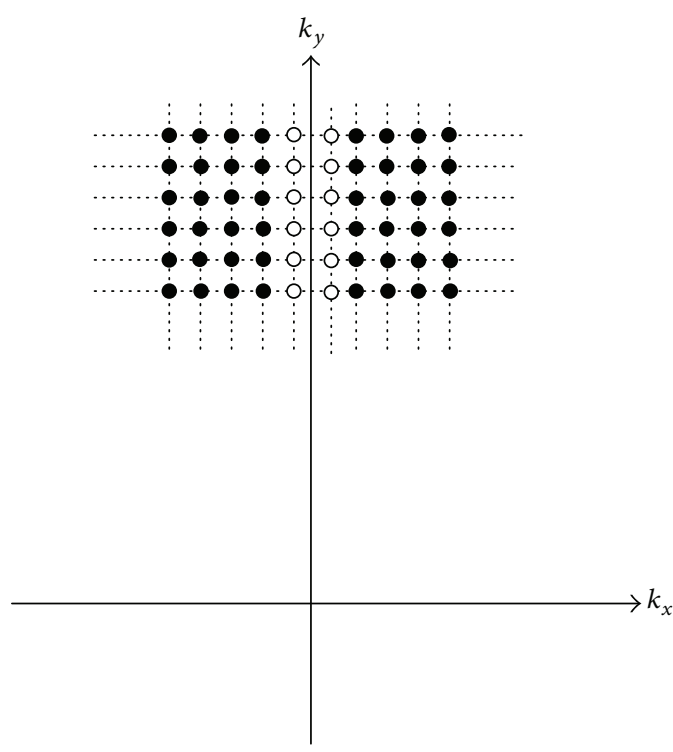

- Sample data of original spatial spectrum O Missed sample data of spatial spectrum

(a)

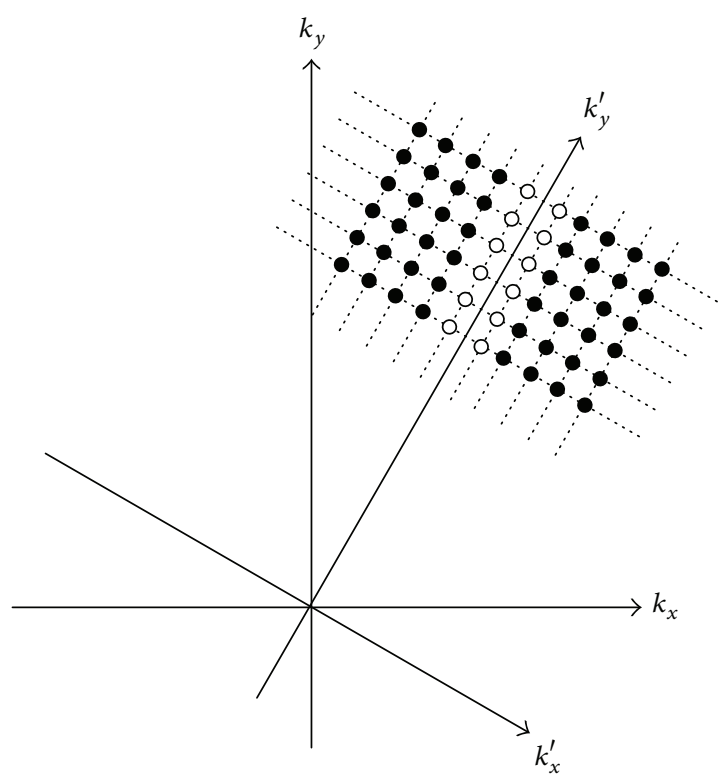

Sample data of original spatial spectrum ○ Missed sample data of spatial spectrum

(b)

Figure 10: The missing sample data of the spatial spectrum can be estimated by the existing data. (a) The target is in the middle line of the array. (b) The target is not in the middle line of the array. In this situation, a transfer matrix is needed.

Step 2. Take the 2D Fourier transform of the imaging result, $I(x, y)$. Then, the corresponding support area of the spatial spectrum is obtained:

$$
E\left(k_{x}, k_{y}\right)=\iint I(x, y) \exp \left[-j\left(k_{x} x+k_{y} y\right)\right] d x d y
$$

Here, $E\left(k_{x}, k_{y}\right)$ is the obtained support area of the spatial spectrum.

Step 3. Extrapolate the missing spatial spectrum. As shown in Figure 10, the black points denote the sample data in the original spatial spectrum and the white points are the missing spatial spectrum. Therefore, the missing spatial spectrum can be forecasted by the existing data. To obtain an accurate result, the left and right data are used to make forward and back forecasting, respectively. The forward forecasting and the back forecasting AR models are given in (18) and (19), respectively:

$$
\begin{aligned}
& E_{L}\left(k_{x, p}, k_{y, q_{0}}\right)=-\sum_{i=1}^{L} b_{L}(i) E\left(k_{x, p-i}, k_{y, q_{0}}\right), \\
& E_{H}\left(k_{x, p}, k_{y, q_{0}}\right)=-\sum_{i=1}^{H} b_{H}(i) E\left(k_{x, p+i}, k_{y, q_{0}}\right),
\end{aligned}
$$

where the variables $L$ and $H$ denote the order of each model. The variables $b_{L}(i)$ and $b_{H}(i)$ denote the coefficient in the models. Here, the model order is evaluated by the forecasting error, and the model coefficient is computed by the Burg algorithm.

Step 4. Update the $k_{y, q_{0}}$ to estimate the missing spatial spectrum. Then, the filled spatial spectrum, denoted by $E^{\prime}\left(k_{x}, k_{y}\right)$, is obtained.

Step 5. Take the 2D inverse Fourier transform of $E^{\prime}\left(k_{x}, k_{y}\right)$. Then, the enhanced image, $I^{\prime}(x, y)$, is obtained:

$$
I^{\prime}(x, y)=\iint E^{\prime}\left(k_{x}, k_{y}\right) \exp \left[j\left(k_{x} x+k_{y} y\right)\right] d k_{x} d k_{y} .
$$

When the target is not in the middle line of the array, the spatial spectrum will not be symmetrical along $k_{y}$ but will be symmetrical along $k_{v}$ (see Figure 2). In this situation, (18) and (19) need to be reconstructed in a new coordinate system. By using the transfer matrix, the variables $k_{x}$ and $k_{y}$ in these expressions are replaced by $k_{x}^{\prime}$ and $k_{y}^{\prime}$ in the new coordinate system. The transfer matrix is

$$
\left[\begin{array}{l}
k_{x}^{\prime} \\
k_{y}^{\prime}
\end{array}\right]=\left[\begin{array}{cc}
\sin \frac{\theta_{t}+\theta_{r}}{2} & -\cos \frac{\theta_{t}+\theta_{r}}{2} \\
-\cos \frac{\theta_{t}^{2}+\theta_{r}}{2} & \sin \frac{\theta_{t}+\theta_{r}}{2}
\end{array}\right]\left[\begin{array}{l}
k_{x} \\
k_{y}
\end{array}\right],
$$

where $\left(\theta_{t}+\theta_{r}\right) / 2$ is the angle of vector $k_{v}$, which is marked in Figure 2.

Additionally, in TWI MIMO radar applications, different targets have different incident angles and received angles. 


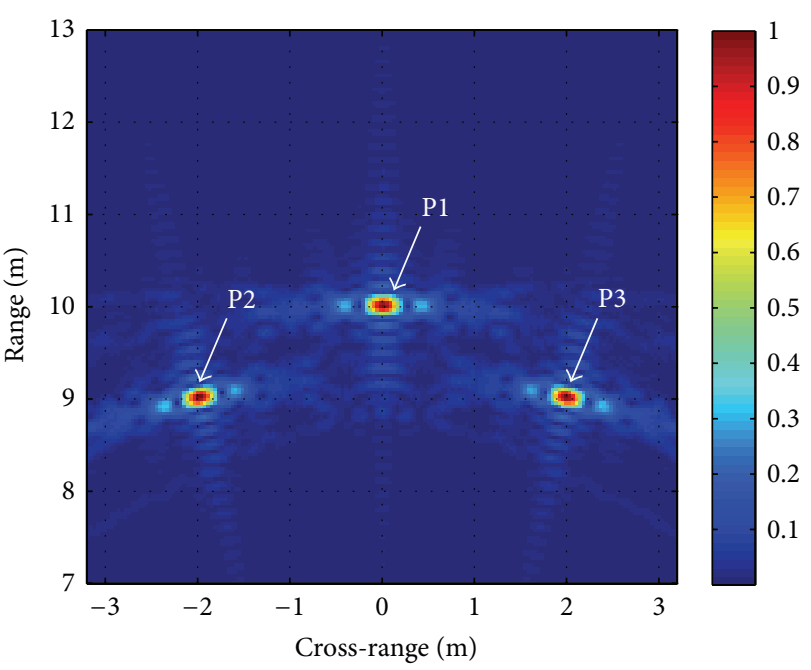

FIGURE 11: The original imaging result by the back projection imaging method.

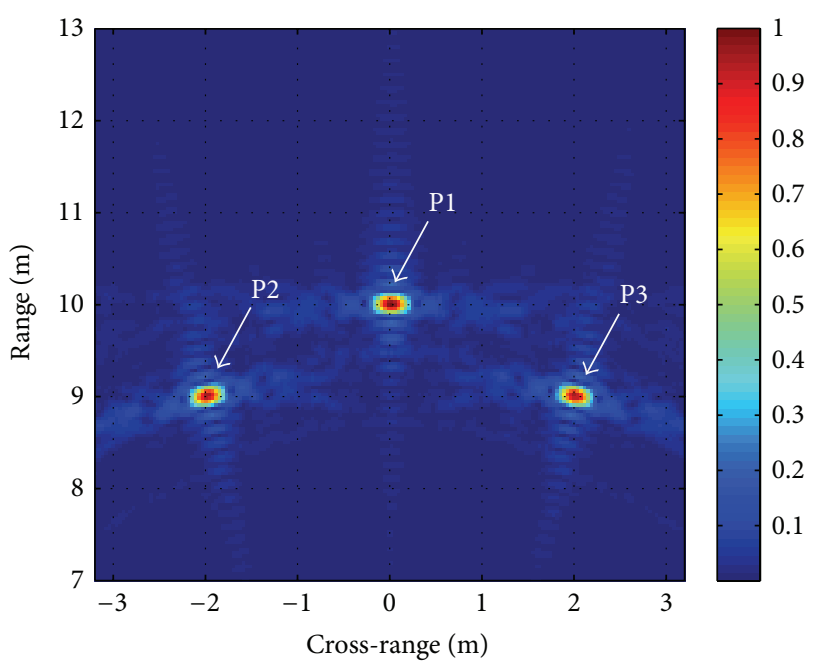

FIGURE 12: The processed result by the proposed method.

Therefore, the spatial spectrums of the targets will be located at different places. To minimize the effects of the overlapped spectrum, the whole image area can be divided into several small subregions. Then, the extrapolation can be applied in each subregion.

\section{Simulated and Experimental Results}

The simulation and experiments used to validate the proposed method are described in this section. In the simulation, the abovementioned array, which is shown in Figure 6, is adopted. In the coordinate system, the $\mathrm{x}$-axis is along the array and the origin is the middle point of the array. Three point targets, located at $(0 \mathrm{~m}, 10 \mathrm{~m}),(-2 \mathrm{~m}, 9 \mathrm{~m})$, and $(2 \mathrm{~m}$, $9 \mathrm{~m})$, are in free space. The stepped frequency signal, with a range from $1 \mathrm{GHz}$ to $2 \mathrm{GHz}$, is used. The increment frequency is $5 \mathrm{MHz}$.

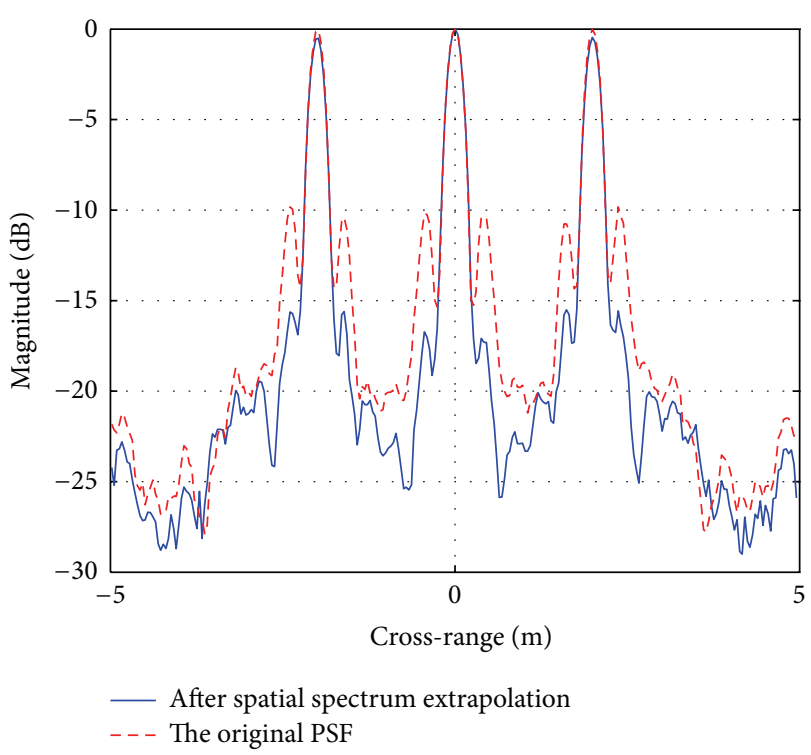

FIGURE 13: The sidelobes are suppressed by the proposed method. The PSLR for each target decreased from $-10.0 \mathrm{~dB}$ to $-15.9 \mathrm{~dB}$, $-17.1 \mathrm{~dB}$, and $-15.9 \mathrm{~dB}$, respectively. The main lobes in this figure are all unchanged.

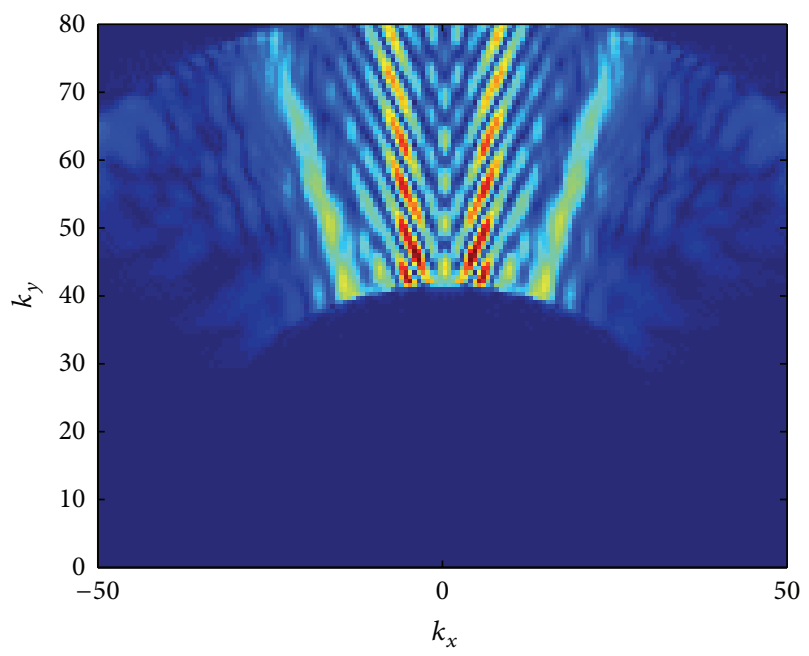

FIGURE 14: The spatial spectrums for the three targets overlap largely when we take the 2D Fourier transform of the whole image area.

Using the back projection (BP) imaging method, the original image is given in Figure 11. Figure 12 is the processed result made by our proposed method. The sidelobes of the three targets are shown to be efficiently suppressed. To analyze the algorithm performance quantitatively, the peak sidelobe ratio (PSLR) for each target is computed, which are suppressed from $-10.0 \mathrm{~dB}$ to $-15.9 \mathrm{~dB},-17.1 \mathrm{~dB}$, and $-15.9 \mathrm{~dB}$ (see Figure 13). The main lobes for the three targets are unchanged.

Because the spatial spectrums of the targets overlap (see Figure 14), in our processing, we divide the imaging area into several subregions. Therefore, the spatial spectrum for 


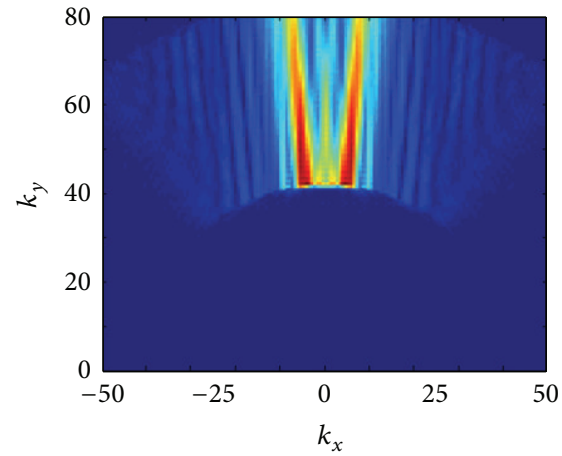

(a)

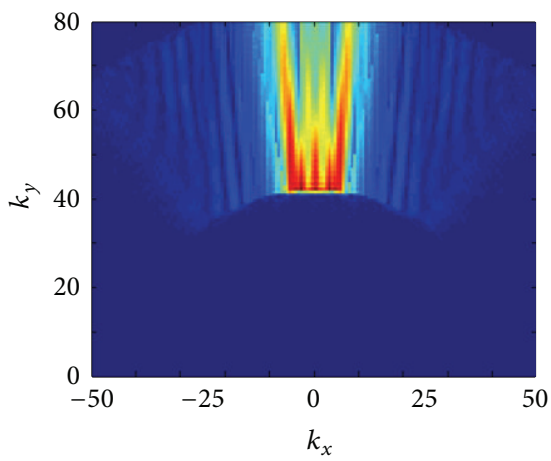

(d)

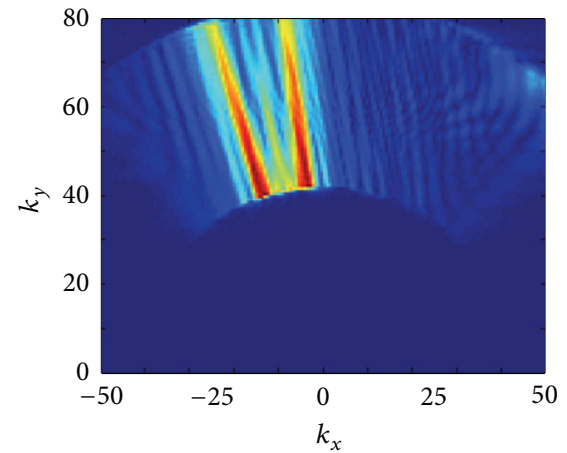

(b)

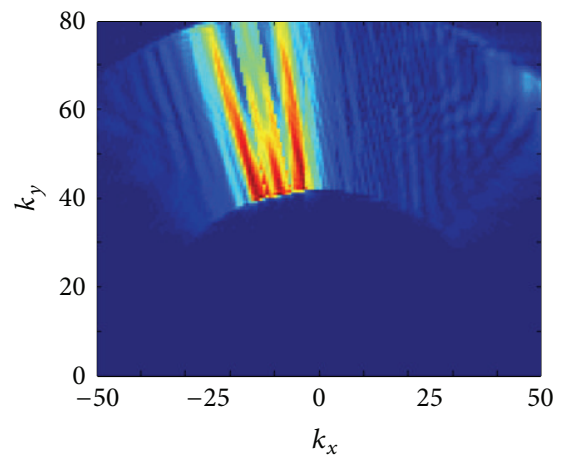

(e)

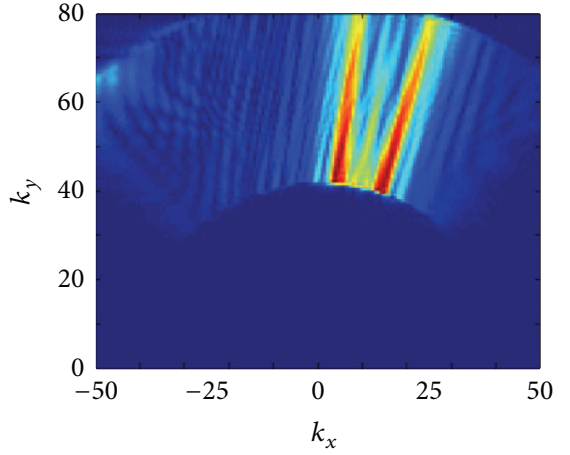

(c)

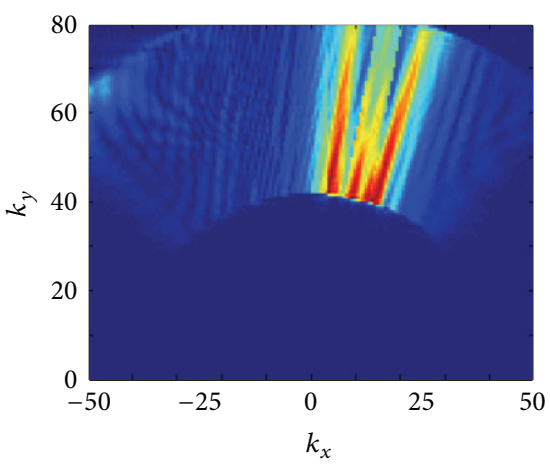

(f)

FIGURE 15: By dividing the imaging area into several subregions, the spatial spectrum for each target is obtained. (a-c) The gapped spatial spectrum for three targets, namely, P1, P2, and P3, respectively. (e-f) The extrapolated spatial spectrum for each target, that is, P1, P2, and P3.

each target is obtained by the 2D Fourier transform, which is shown in Figure 15. From Figures 15(a)-15(c), the spatial spectrums for these three targets are shown to be gapped. After spatial spectrum estimation, the missing spectrums are filled in (see Figures 15(d)-15(f)).

To validate the performance of the proposed method, the through-the-wall imaging experiments are processed in a real environment. In this experiment, a sparse STVA array, which has two transmitters and six receivers, is used. The length of the array is $4.1 \mathrm{~m}$, and two transmitters are placed at the two ends of the array. The height of the array is $1.5 \mathrm{~m}$ and the interelement space of the receivers is $0.25 \mathrm{~m}$. The antennas used in the system are Archimedes antennas. A transreceiver module is designed to transmit and receive the EM wave. The waveform used in the system is stepped frequency signal. Its frequency range is from $1 \mathrm{GHz}$ to $2 \mathrm{GHz}$, with the increment step of $2 \mathrm{MHz}$. The principle of the radar system is given in Figure 16.

Imaging data were collected by the radar system. Radar system is placed at the left side of a cinderblock building at a distance of $23.7 \mathrm{~m}$. The antennas are parallel to the side wall. As shown in Figure 17, the thickness of the left-side cinderblock wall is $30 \mathrm{~cm}$. The width of the left wall is $10 \mathrm{~m}$ and its extent is $4 \mathrm{~m}$ of the first floor. During the acquisition of the data, a 172-cm-tall man stands behind the wall and moves slightly (see Figure 17).
A standard differential back projection (BP) imaging algorithm is adopted to process the acquired data. It is noted that the wall parameters are estimated and compensated by using the image-domain method (see [14] for details). Figure 18 is the original imaging result. The human imaging has strong sidelobes and the image quality is severely affected. Using the proposed method, the processed result is shown in Figure 19. Compared with Figure 18, the sidelobes are efficiently suppressed and the image quality is significantly enhanced.

To validate the algorithm performance for the static target behind the wall, another experiment is processed. In the experiment, we use a $30 \mathrm{~cm}$ trihedral as the target. The trihedral is placed in the abovementioned building, with $2 \mathrm{~m}$ behind the side cinderblock wall (see Figure 20). The radar system is placed at the left side of the building, with a distance of $9 \mathrm{~m}$.

By using the BP imaging method and background subtract technique, the original imaging result is obtained (see Figure 21). As shown in the figure, the trihedral is present in the image, but it has strong sidelobes. Then we use the proposed method to extrapolate its spatial spectrum, and consequently, the processing result is obtained (see Figure 22). It is shown that, after processed by the proposed method, the target imaging is significantly enhanced and its sidelobes are suppressed. 


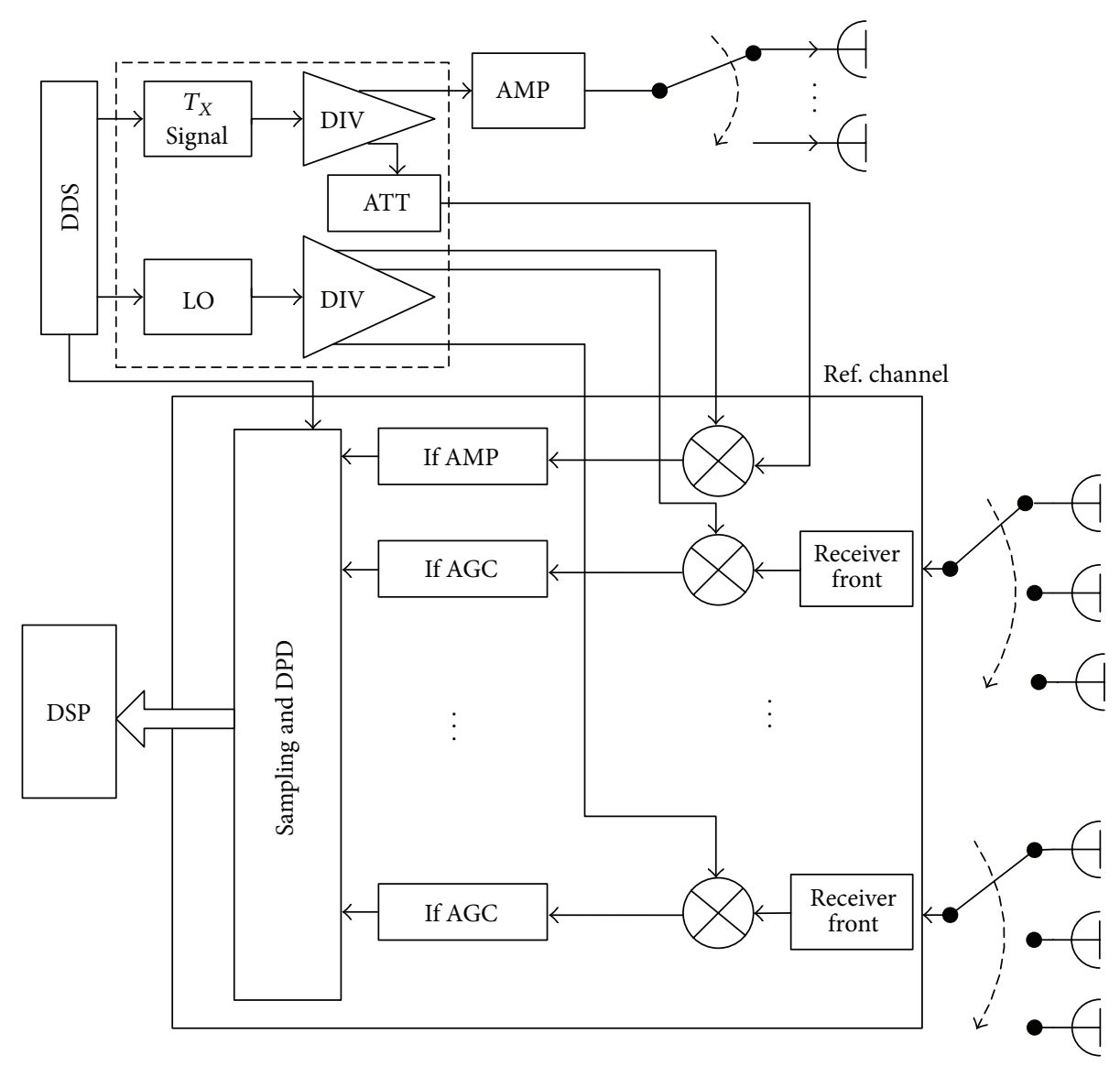

FIGURE 16: The principle diagram of through-the-wall radar system.

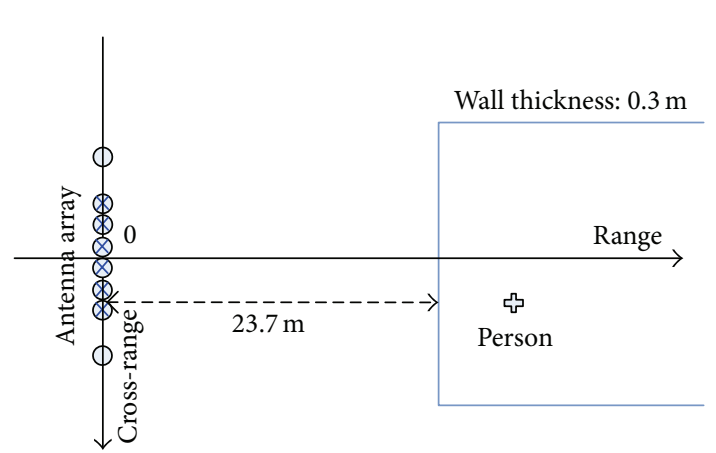

(a)

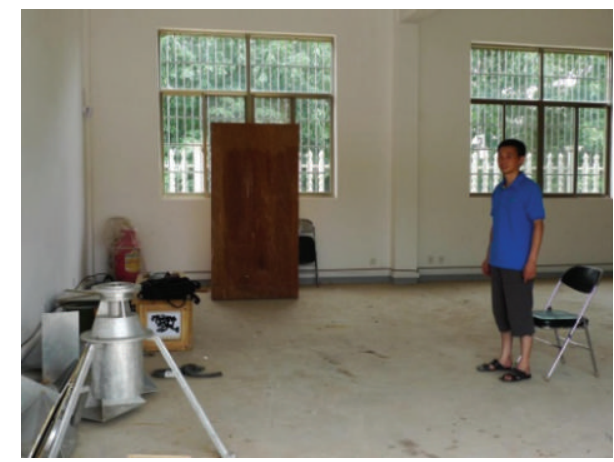

(b)

FIGURE 17: Imaging of a person inside a building: (a) the geometry of the imaging and (b) a man stands behind the left-side cinderblock wall.

\section{Conclusion}

In this paper, we construct an imaging model for an UWB MIMO radar via the spatial spectrum. The rigorous derivation of the model shows that the more spatial spectrum is used, the better imaging performance will be obtained. Therefore, when designing a MIMO array, the best solution is to make full use of the spatial spectrum. Unfortunately, to keep the system complexity at a reasonable level and conform to the propagation demands, MIMO arrays are usually sparse in through-the-wall applications, which results in corrupted and gapped data. The corresponding imaging results are seriously affected by the high-level sidelobes.

Aiming at this problem, we proposed a spatial spectrumbased imaging enhancement method in this paper. By estimating the missing spatial spectrum, the effects of the gapped virtual elements can be significantly minimized. The processing results of the synthetic and experimental data show that the proposed method can efficiently improve the 


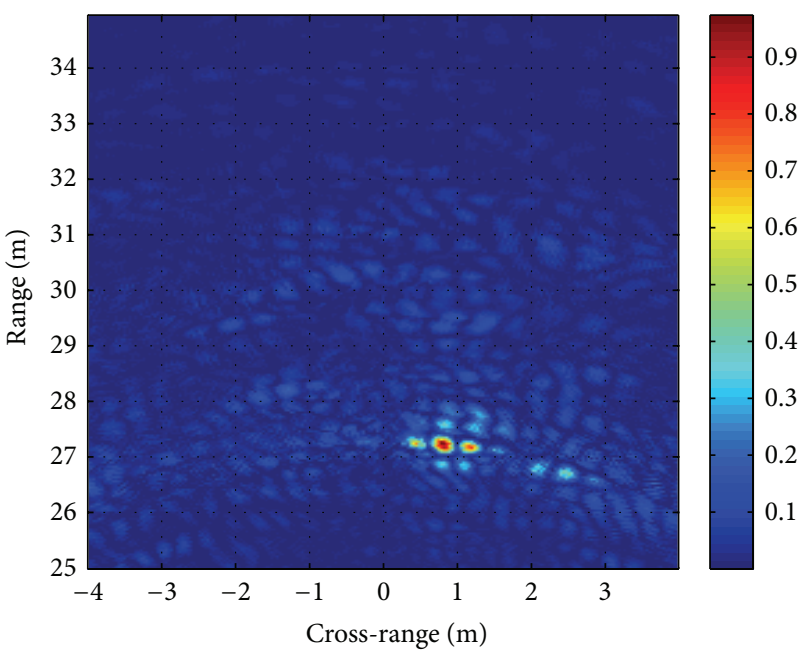

FIGURE 18: The original imaging result by the standard differential BP imaging algorithm.

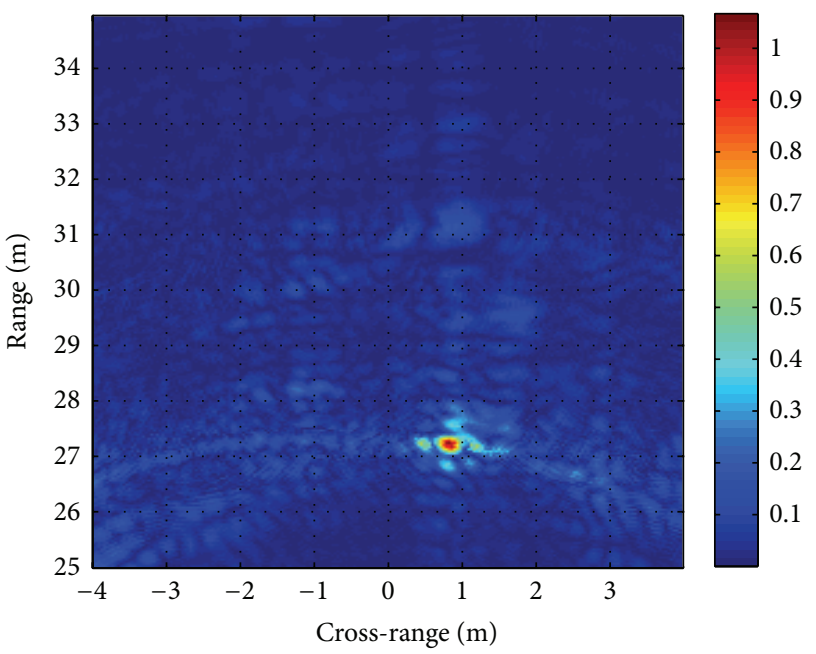

FIGURE 19: The processing result by the method proposed in this paper.

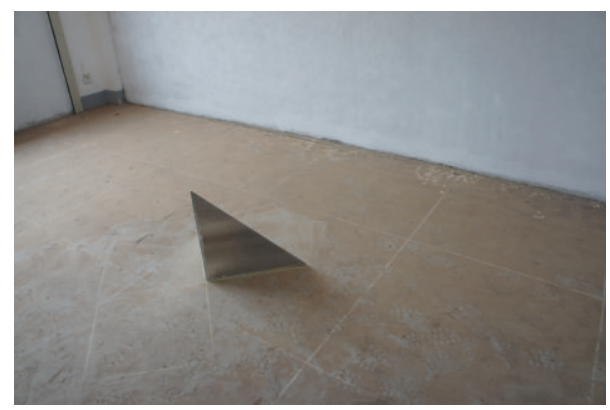

Figure 20: In the static target experiment, a trihedral is used as the target and placed $2 \mathrm{~m}$ behind the wall.

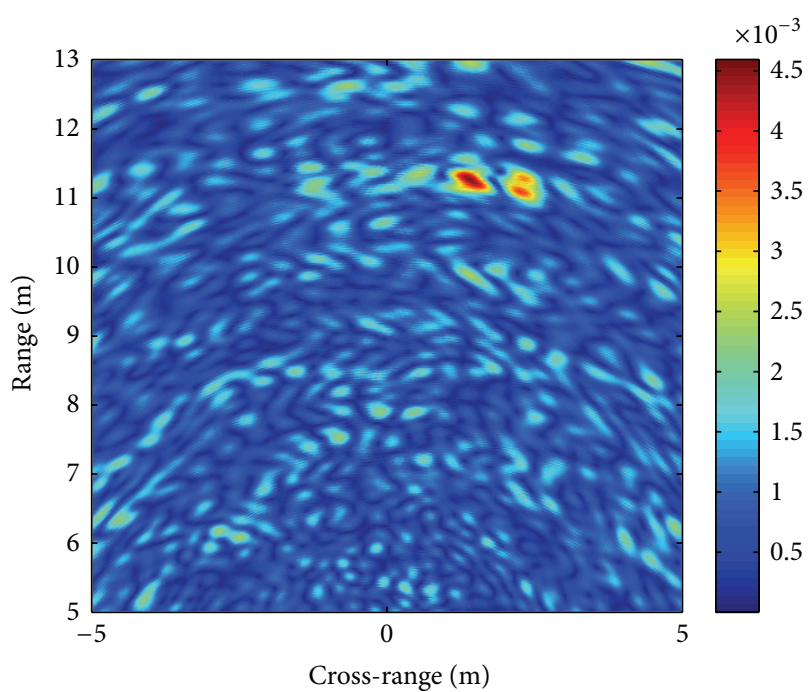

FIgURE 21: The original imaging result of the trihedral. In the figure, target has strong sidelobes.

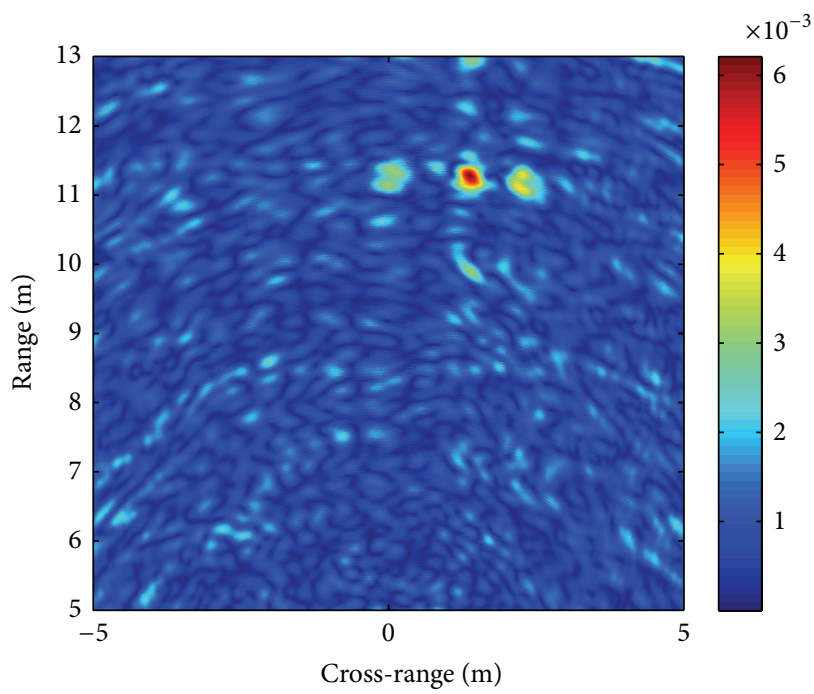

FIGURE 22: By using the proposed method, the sidelobes are suppressed.

imaging quality for both the moving target and static target in through-the-wall applications. Unlike the conventional amplitude weighting methods and nonlinear techniques, the proposed method does not degrade the main lobe resolution when suppressing the sidelobes. At present, the proposed method has been successfully applied to our real throughthe-wall radar system.

\section{Conflict of Interests}

The authors declare that there is no conflict of interests regarding the publication of this paper. 


\section{Acknowledgment}

This work was supported in part by the National Natural Science Foundation of China under Grants 61372161 and 61271441.

\section{References}

[1] M. G. Amin, Through-the-Wall Radar Imaging, CRC press, 2010.

[2] G. E. Smith and B. G. Mobasseri, "Robust through-the-wall radar image classification using a target-model alignment procedure," IEEE Transactions on Image Processing, vol. 21, no. 2, pp. 754-767, 2012.

[3] V. H. Tang, A. Bouzerdoum, and S. L. Phung, "Two-stage through-the-wall radar image formation using compressive sensing," Journal of Electronic Imaging, vol. 22, no. 2, pp. 1-15, 2013.

[4] K. E. Browne, R. J. Burkholder, and J. L. Volakis, "Fast optimization of through-wall radar images via the method of Lagrange multipliers," IEEE Transactions on Antennas and Propagation, vol. 61, no. 1, pp. 320-328, 2013.

[5] C. Debes, A. M. Zoubir, and M. G. Amin, "Enhanced detection using target polarization signatures in through-the-wall radar imaging," IEEE Transactions on Geoscience and Remote Sensing, vol. 50, no. 5, pp. 1968-1979, 2012.

[6] F. Ahmad and M. G. Amin, "Through-the-wall human motion indication using sparsity-driven change detection," IEEE Transactions on Geoscience and Remote Sensing, vol. 51, no. 3, pp. 881$890,2013$.

[7] M. Dehmollaian and K. Sarabandi, "Refocusing through building walls using synthetic aperture radar," IEEE Transactions on Geoscience and Remote Sensing, vol. 46, no. 6, pp. 1589-1599, 2008.

[8] A. H. Muqaibel and A. Safaai-Jazi, "A new formulation for characterization of materials based on measured insertion transfer function," IEEE Transactions on Microwave Theory and Techniques, vol. 51, no. 8, pp. 1946-1951, 2003.

[9] H. Wang, Z. O. Zhou, and L. J. Kong, "Wall parameters estimation for moving target localization with through-thewall radar," in Proceedings of the International Conference on Microwave and Millimeter Wave Technology (ICMMT '07), pp. 1-4, IEEE Computer Society, Guilin, China, 2007.

[10] X. Li, D. X. An, X. T. Huang, and S. R. Peng, "Estimation of wall parameters based on range profiles," Science China Information Sciences, vol. 54, no. 10, pp. 2178-2189, 2011.

[11] W.-Q. Wang, "Virtual antenna array analysis for MIMO synthetic aperture radars," International Journal of Antennas and Propagation, vol. 2012, Article ID 587276, 10 pages, 2012.

[12] J. L. Schwartz and B. D. Steinberg, "Ultrasparse, ultrawideband arrays," IEEE Transactions on Ultrasonics, Ferroelectrics, and Frequency Control, vol. 45, no. 2, pp. 376-393, 1998.

[13] M. Mohsin Riaz and A. Ghafoor, "Through-wall image enhancement based on singular value decomposition," International Journal of Antennas and Propagation, vol. 2012, Article ID 961829, 20 pages, 2012.

[14] T. Jin, B. Chen, and Z. Zhou, "Image-domain estimation of wall parameters for autofocusing of through-the-wall SAR imagery," IEEE Transactions on Geoscience and Remote Sensing, vol. 51, no. 3, pp. 1836-1843, 2013.
[15] F. Ahmad, M. G. Amin, and G. Mandapati, "Autofocusing of through-the-wall radar imagery under unknown wall characteristics," IEEE Transactions on Image Processing, vol. 16, no. 7, pp. 1785-1795, 2007.

[16] F. Ahmad, Y. Zhang, and M. G. Amin, "Three-dimensional wideband beamforming for imaging through a single wall," IEEE Geoscience and Remote Sensing Letters, vol. 5, no. 2, pp. 176-179, 2008.

[17] F. Soldovieri, R. Solimene, and R. Pierri, "A simple strategy to detect changes in through the wall imaging," Progress In Electromagnetics Research M, vol. 7, pp. 1-13, 2009.

[18] W. Zhang and A. Hoorfar, "Three-dimensional real-time through-the-wall radar imaging with diffraction tomographic algorithm," IEEE Transactions on Geoscience and Remote Sensing, vol. 51, no. 7, pp. 4155-4163, 2013.

[19] H. C. Stankwitz, R. J. Dallaire, and J. R. Fienup, "Nonlinear apodization for sidelobe control in SAR imagery," IEEE Transactions on Aerospace and Electronic Systems, vol. 31, no. 1, pp. 267-279, 1995.

[20] T. K. Sjögren, V. T. Vu, and M. I. Pettersson, "2D apodization in UWB SAR using linear filtering," in Proceedings of the IEEE International Geoscience and Remote Sensing Symposium (IGARSS '11), pp. 1689-1692, Vancouver, Canada, July 2011.

[21] X. Zhuge, A. G. Yarovoy, and L. P. Ligthart, "A sidelobe reduction technique for enhancing images of UWB sparse MIMO array," in Proceedings of the International Radar ConferenceSurveillance for a Safer World, pp. 1-6, 2009.

[22] H. C. Stankwitz and M. R. Kosek, "Sparse aperture fill for SAR using super-SVA," in Proceedings of the IEEE National Radar Conference, pp. 70-75, Ann Arbor, Mich, USA, May 1996.

[23] L. Wang, X. Huang, Z. Zhimin, and S. Xiaokun, "Control sidelobes in UWB SAR images," in Proceedings of the IEEE International Geoscience and Remote Sensing Symposium, pp. 4630-4632, Seoul, The Republic of Korea, July 2005.

[24] R. Goodman, S. Tummala, and W. Carrara, "Issues in ultrawideband, widebeam SAR image formation," in Proceedings of the IEEE International Radar Conference, pp. 479-485, Alexandria, VA, USA, May 1995.

[25] K. E. Browne, R. J. Burkholder, and J. L. Volakis, “Throughwall opportunistic sensing system utilizing a low-cost flat-panel array," IEEE Transactions on Antennas and Propagation, vol. 59, no. 3, pp. 859-868, 2011.

[26] E. G. Larsson, P. Stoica, and J. Li, "Amplitude spectrum estimation for two-dimensional gapped data," IEEE Transactions on Signal Processing, vol. 50, no. 6, pp. 1343-1354, 2002.

[27] B. Lu, Y. Zhao, X. Sun, and Z. Zhou, "Design and analysis of ultra wide band split transmit virtual aperture array for through the wall imaging," International Journal of Antennas and Propagation, vol. 2013, Article ID 934509, 9 pages, 2013.

[28] H. J. Wang, Y. Su, Y. T. Zhu, and H. B. Xu, "MIMO radar imaging based on spatial spectral-domain filling," Acta Electronica Sinica, vol. 36, no. 6, pp. 1242-1246, 2009. 

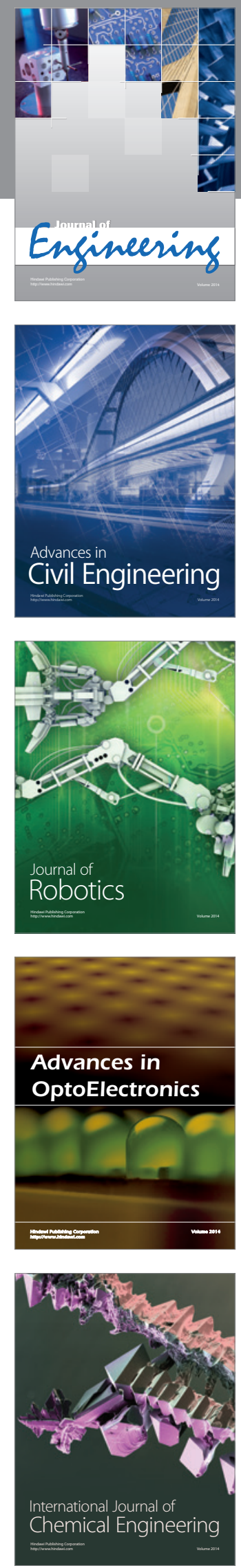

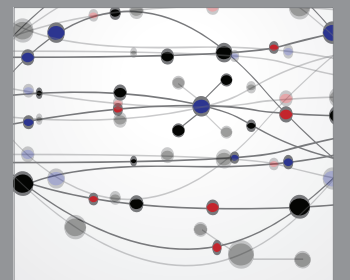

The Scientific World Journal
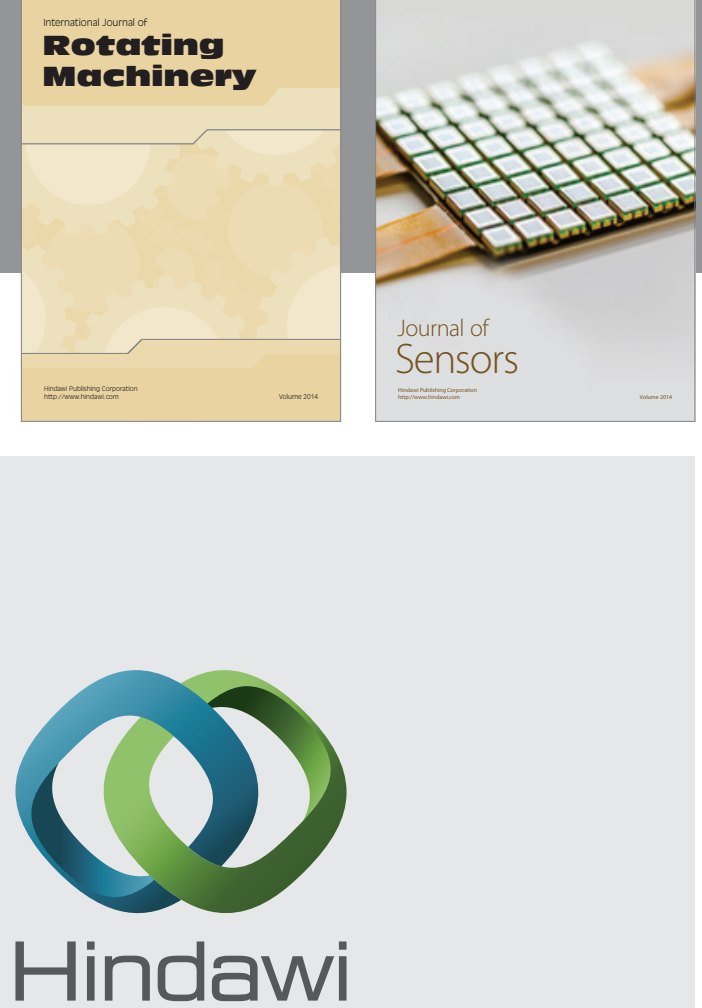

Submit your manuscripts at http://www.hindawi.com
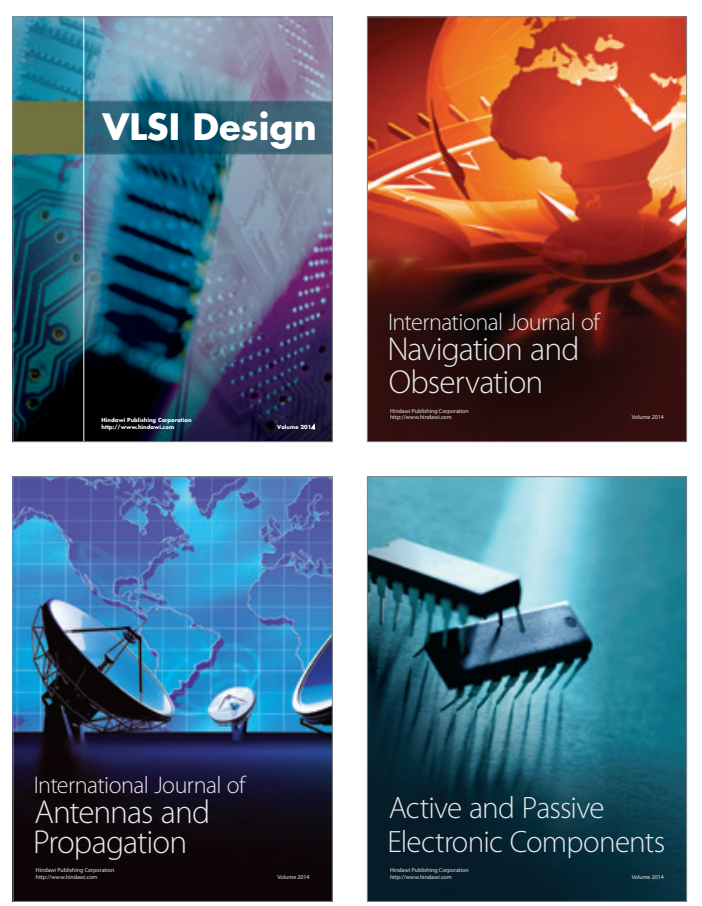
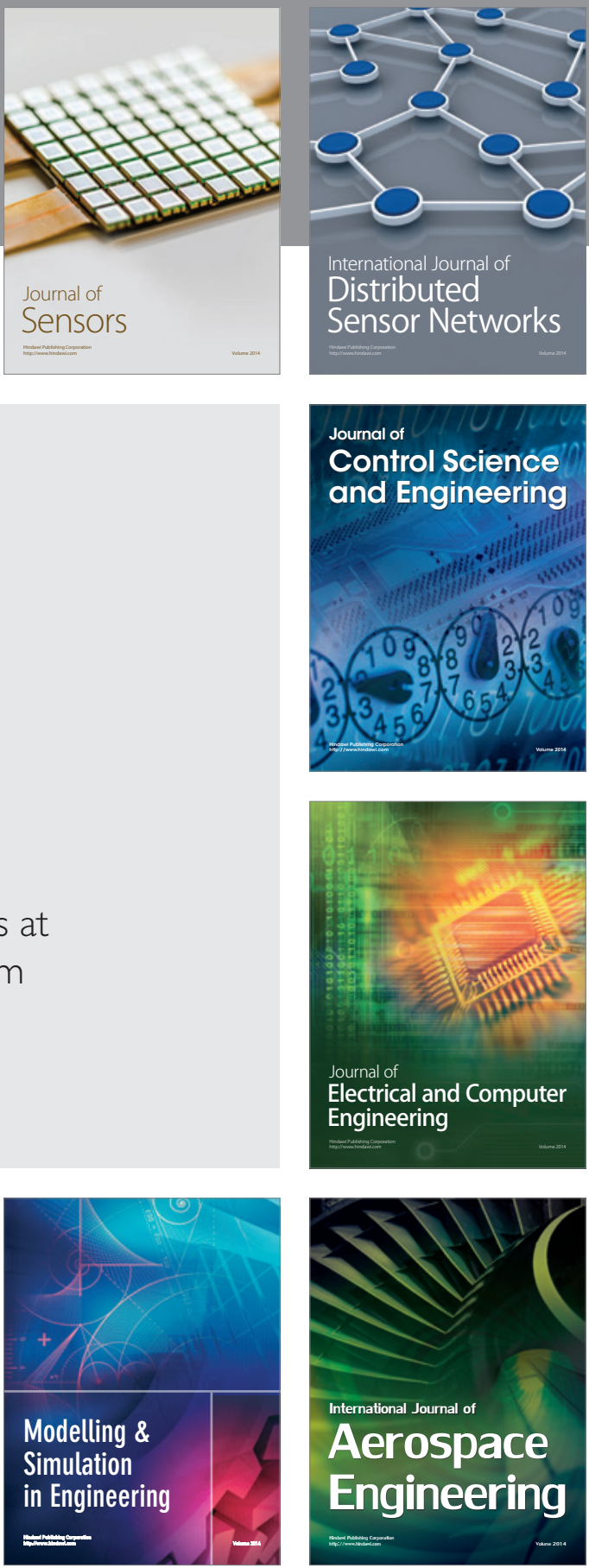

Journal of

Control Science

and Engineering
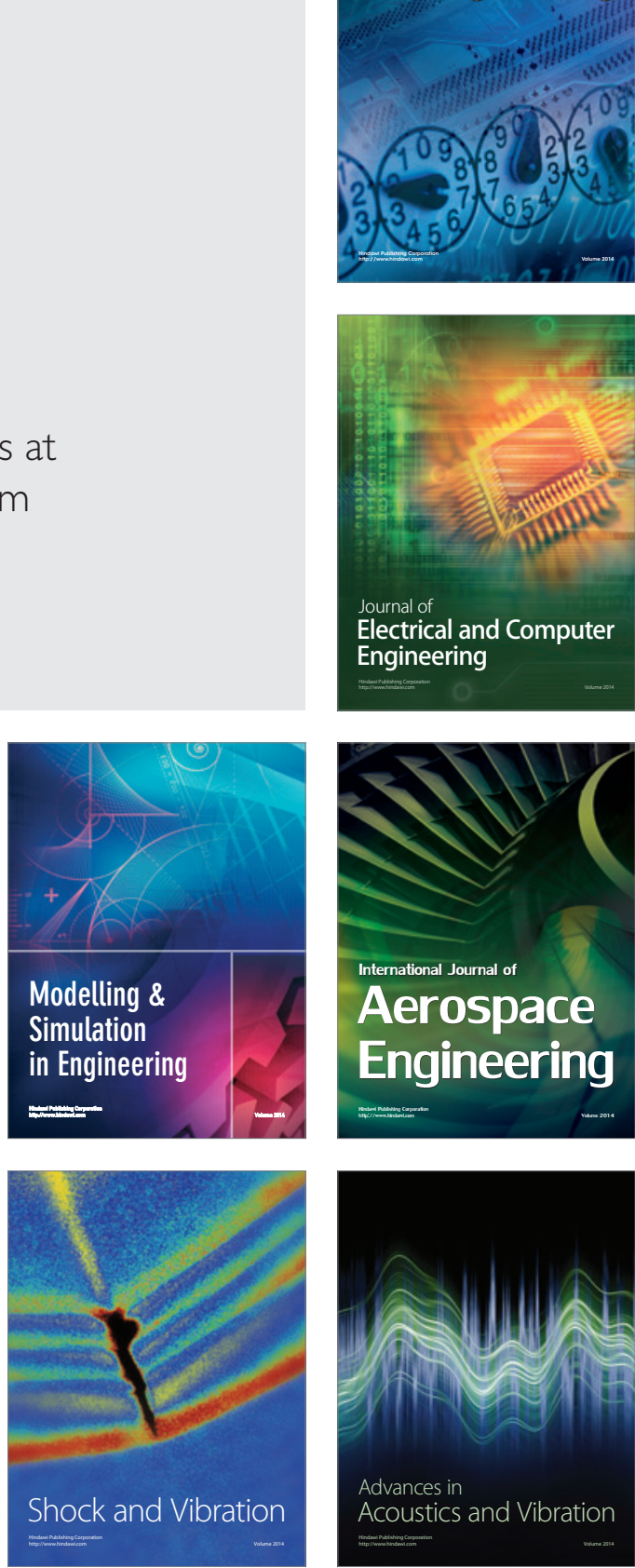